IPMU-12-0232

October 17, 2018

\title{
Model Independent Analysis of Interactions between Dark Matter and Various Quarks
}

\author{
Biplob Bhattacherjee ${ }^{(a)}$, Debajyoti Choudhury ${ }^{(b)}$, Keisuke Harigaya $^{(a)}$, \\ Shigeki Matsumoto ${ }^{(a)}$ and Mihoko M. Nojiri ${ }^{(c, a)}$ \\ ${ }^{(a)}$ Kavli IPMU, University of Tokyo, Kashiwa, 277-8583, Japan \\ (b) Department of Physics and Astrophysics, \\ University of Delhi, Delhi 110007, India. \\ (c) Theory Group, KEK, Tsukuba, 305-0801, Japan
}

\begin{abstract}
Present and future expected limits on interactions between dark matter and various quarks are thoroughly investigated in a model-independent way. In particular, the constraints on the interactions from the Large Hadron Collider (LHC) experiment are carefully considered with a focus on mono jet + missing transverse energy $\left(\mathbb{E}_{T}\right)$, mono b-jet $+\mathbb{E}_{T}$, and top quark(s) $+\mathbb{E}_{T}$ channels. Model-independent upper limits (expected limits) on the cross section times acceptance for non-standard model events are derived for the LHC operating at $7 / 8 / 14 \mathrm{TeVs}$. Assuming that the dark matter is a singlet real scalar or a singlet Majorana fermion, we also put constraints on several operators describing its interactions with up, down, strange, charm, bottom and top quarks. These constraints are compared to those obtained from cosmological and astrophysical implications.
\end{abstract}




\section{Introduction}

The origin of the dark matter in the Universe and its nature constitute some of the most outstanding mysteries in modern particle physics, cosmology, and astrophysics. Though the problem is not resolved yet, many efforts have been made over the years (and continue to be made) in the search for dark matter (DM), and these give precious information about several DM interactions. Theoretically, the weakly interacting massive particle (WIMP), called so as its couplings are of the order of the electroweak (EW) couplings, is one of the promising candidates for dark matter [1]. Experimentally, direct detection experiments of DM put very severe limits on the scattering cross section between the DM and a nucleon [2, while indirect detection experiments give constraints on self-annihilation cross sections of the DM into various final states [3].

Among DM interactions, those to quarks and gluons are particularly well investigated thanks to the Large Hadron Collider (LHC) experiment. Importantly, the LHC experiment enables us to explore not only the interactions relevant to direct and indirect DM detections but also certain interactions irrelevant to those detections. Consequently, many studies have already been performed assuming a definite relation among the DM interactions with various quarks or using an analysis with simple collider simulations [4]. In this article, we thoroughly investigate present and (near) future expected limits on the DM interaction with individual quark flavours, focussing on mono jet + missing transverse energy $\left(\mathbb{E}_{T}\right)$, mono b-jet $+\mathbb{E}_{T}$, and top quark(s) $+\mathbb{E}_{T}$ channels, which are all derived from dedicated collider simulations using a model-independent method.

In the next section (section 2), we first summarize DM interactions assuming that the DM particle is a real scalar or a Majorana fermion and singlet under SM gauge

groups2. Such candidates are frequently used in the literature and can be regarded as the simplest examples of a DM particle. We then put limits on the operators from

\footnotetext{
${ }^{1}$ The DM interaction with gluon can also be investigated by the LHC experiment and severe constraints are obtained in past works 4 .

${ }^{2}$ The requirement of being singlet is not strictly necessary, but a simplifying assumption. A charged DM in the sky would interact with cosmic rays (impeding their propagation), as well as the CMB photons (thereby distorting the blackbody spectrum beyond permissible limits). Similarly, neutral but coloured DM would be captured by nuclei to lead to exotic isotopes. A prominent counterexample of a neutral, but gauge nonsinglet DM is given by the lightest neutralino in the minimal supersymmetric standard model.
} 
cosmological and astrophysical observations of the DM in section 3. We next consider what kinds of DM signals are generally expected at the LHC experiment in section 4 and give model-independent upper limits (expected limits) on the cross section times acceptance for DM signals at $7 \mathrm{TeV}$ ( 8 or $14 \mathrm{TeV}$ ) run. It turns out that these limits are very useful to constrain any DM quark interactions. Using the obtained limits, we also explain how to put a limit on each operator (discussed in section 2) describing a DM interaction with various quarks. We finally consider, in section 5. how severe limits can the LHC experiment put onsuch operators and compare with those obtained from cosmological and astrophysical observations. Section 6 is devoted to a summary of our discussions.

\section{Dark matter interactions}

Dark matter interactions with various quarks are discussed in this section assuming that the DM is a real scalar or a Majorana fermion which is singlet under SM gauge groups. We introduce operators describing interactions between the DM and SM particles up to mass-dimension six. Decoupling and/or weakly coupled heavy physics behind the interactions are implicitly assumed, so that the whole theory (i.e., the ultraviolet completion) is renormalizable. As the stability of the DM is guaranteed by imposing a $Z_{2}$ symmetry (under which the DM field is odd, while all the SM fields are even), the operators have to involve two DM fields. The real scalar DM is denoted by $\phi$, while the Majorana fermion DM is denoted by $\chi$ in the following discussions.

The effective lagrangian for the scalar DM field $\phi$ is simply given by

$$
\mathcal{L}_{\mathrm{eff}}^{(\phi)}=\mathcal{L}_{\mathrm{SM}}+\left[\frac{1}{2}(\partial \phi)^{2}-\frac{M_{\phi}^{2}}{2} \phi^{2}\right]+\sum_{n=4}^{\infty} \frac{1}{\Lambda^{n-4}}\left[\sum_{i} c_{i} \mathcal{O}_{i}^{(n)}+\text { h.c. }\right]
$$

where $\mathcal{L}_{\mathrm{SM}}$ is the SM lagrangian. Since several interactions contribute to the DM mass after the electroweak symmetry breaking, the physical DM $m_{\phi}$ (we use this notation throughout the paper) is distinct from $M_{\phi}$. The mass dimension of operator

$\mathcal{O}_{i}^{(n)}$ is denoted by $n$, while $\Lambda$ is the cutoff scale below which the effective lagrangian describes the physics. The complete set of operators upto dimension six, which are 
invariant under both Lorentz group and SM gauge groups, is given by

$$
\begin{array}{ll}
\mathcal{O}_{\phi H}^{(4)}=\phi^{2}|H|^{2}, & \mathcal{O}_{\phi L H E}^{(6)}=\phi^{2} \bar{L} H E \\
\mathcal{O}_{\phi H}^{(6)}=\phi^{2}|H|^{4}, & \mathcal{O}_{\phi Q H D}^{(6)}=\phi^{2} \bar{Q} H D \\
\mathcal{O}_{\phi \partial H}^{(6)}=\phi^{2}\left|D_{\mu} H\right|^{2}, & \mathcal{O}_{\phi Q H U}^{(6)}=\phi^{2} \bar{Q} H^{c} U, \\
\mathcal{O}_{\phi V V}^{(6)}=\phi^{2} V_{\mu \nu} V^{\mu \nu}, & \mathcal{O}_{\phi V \tilde{V}}^{(6)}=\phi^{2} V_{\mu \nu} \tilde{V}^{\mu \nu}
\end{array}
$$

where $H, L=\left(\nu, e_{L}\right)^{T}, E, Q=\left(u_{L}, d_{L}\right)^{T}, D$, and $U$ are the higgs doublet, the lepton doublet, the charged lepton singlet, the doublet quark, the down-type singlet quark, and the up-type singlet quark, respectively. On the other hand, $V_{\mu \nu}\left(\tilde{V}_{\mu \nu} \equiv\right.$ $\left.\epsilon_{\mu \nu \alpha \beta} V^{\alpha \beta}\right)$ is the field strength tensor for a gauge boson, namely, $V_{\mu \nu}=B_{\mu \nu}, W_{\mu \nu}^{j}$, and $G_{\mu \nu}^{a}$ are those for the hyper-charge gauge boson, the weak $\mathrm{SU}(2)_{L}$ gauge boson, and the gluon, respectively. The covariant derivative is denoted by $D_{\mu}$. Operators $\mathcal{O}_{\phi L H E}^{(6)}, \mathcal{O}_{\phi Q H D}^{(6)}$, and $\mathcal{O}_{\phi Q H U}^{(6)}$ are in fact flavor-dependent (e.g., $\left.\phi^{2} \bar{Q}_{I} H D_{J}\right)$. In order to avoid dangerous flavor changing processes, we only consider flavor diagonal parts of the operators 3 . In other words, we implicitly assume that heavy physics has some mechanism to suppress flavor off-diagonal parts of the operators.

Since we are interested in interactions between DM and various quarks, we focus on $\mathcal{O}_{\phi Q_{i} H D_{i}}^{(6)}$ and $\mathcal{O}_{\phi Q_{i} H U_{i}}^{(6)}(i=1,2,3)$ among the operators listed above. After the electroweak symmetry breaking $\left(\langle 0|H| 0\rangle=(0, v)^{T} / \sqrt{2}\right.$ with $v$ being about $\left.246 \mathrm{GeV}\right)$, these operators give following effective interactions,

$$
\mathcal{L}_{\text {int }}^{(\phi)}=\frac{v \phi^{2}}{\sqrt{2} \Lambda^{2}} \sum_{i=1}^{3}\left[\bar{u}_{i}\left(c_{\phi Q_{i} H U_{i}}^{(R)}+i c_{\phi Q_{i} H U_{i}}^{(I)} \gamma_{5}\right) u_{i}+\bar{d}_{i}\left(c_{\phi Q_{i} H D_{i}}^{(R)}+i c_{\phi Q_{i} H D_{i}}^{(I)} \gamma_{5}\right) d_{i}\right]
$$

where $c_{\phi Q_{i} H U_{i}}^{(R)}\left(c_{\phi Q_{i} H U_{i}}^{(I)}\right)$ and $c_{\phi Q_{i} H D_{i}}^{(R)}\left(c_{\phi Q_{i} H D_{i}}^{(I)}\right)$ are the real (imaginary) parts of the Wilson coefficients $c_{\phi Q_{i} H U_{i}}$ and $c_{\phi Q_{i} H D_{i}}$ corresponding to the operators $\mathcal{O}_{\phi Q_{i} H U_{i}}^{(6)}$ and $\mathcal{O}_{\phi Q_{i} H D_{i}}^{(6)}$

As in the case of the real scalar DM field $\phi$, the effective lagrangian for the Majorana fermion DM field $\chi$ is also given in the same form,

$$
\mathcal{L}_{\mathrm{eff}}^{(\chi)}=\mathcal{L}_{\mathrm{SM}}+\frac{1}{2} \bar{\chi}\left(i \not \partial-M_{\chi}\right) \chi+\sum_{n=5}^{\infty} \frac{1}{\Lambda^{n-4}}\left[\sum_{i} c_{i} \mathcal{O}_{i}^{(n)}+\text { h.c. }\right],
$$

where we adopt the four component notation for the Majorana field $\chi=\chi^{c}$ with the superscript ' $c$ ' denoting charge conjugation. Once again, the physical mass $m_{\chi}$

\footnotetext{
${ }^{3}$ This is not strictly necessary, though. For example, the leading flavour changing neutral current interaction that a term such as $\phi^{2} \bar{Q}_{3} H^{c} u_{1}$ would generate is the anomalous $\bar{t} u H$ vertex, and that too at one-loop. This clearly is harmless. Nonetheless, we desist from admitting such terms, simply because their inclusion does not result in any qualitative change in DM physics.
} 
is distinct from the mass parameter $M_{\chi}$. The complete set of effective operators involving two DM fields is given by

$$
\begin{array}{ll}
\mathcal{O}_{\chi H, 1}^{(5)}=(\bar{\chi} \chi)|H|^{2}, & \mathcal{O}_{\chi U}^{(6)}=\left(\bar{\chi} \gamma^{\mu} \gamma_{5} \chi\right)\left(\bar{U} \gamma_{\mu} U\right) \\
\mathcal{O}_{\chi H, 2}^{(5)}=i\left(\bar{\chi} \gamma_{5} \chi\right)|H|^{2}, & \mathcal{O}_{\chi D}^{(6)}=\left(\bar{\chi} \gamma^{\mu} \gamma_{5} \chi\right)\left(\bar{D} \gamma_{\mu} D\right) \\
\mathcal{O}_{\chi H}^{(6)}=\left(\bar{\chi} \gamma^{\mu} \gamma_{5} \chi\right)\left(H^{\dagger} i \overleftrightarrow{D_{\mu}} H\right), & \mathcal{O}_{\chi L}^{(6)}=\left(\bar{\chi} \gamma^{\mu} \gamma_{5} \chi\right)\left(\bar{L} \gamma_{\mu} L\right) \\
\mathcal{O}_{\chi Q}^{(6)}=\left(\bar{\chi} \gamma^{\mu} \gamma_{5} \chi\right)\left(\bar{Q} \gamma_{\mu} Q\right), & \mathcal{O}_{\chi E}^{(6)}=\left(\bar{\chi} \gamma^{\mu} \gamma_{5} \chi\right)\left(\bar{E} \gamma_{\mu} E\right)
\end{array}
$$

For the same reason as in the scalar DM case, we focus only on flavor diagonal parts of the operators $\mathcal{O}_{\chi Q}^{(6)}, \mathcal{O}_{\chi U}^{(6)}$, and $\mathcal{O}_{\chi D}^{(6)}$, which lead to

$$
\mathcal{L}_{\text {int }}^{(\chi)}=\frac{2 \bar{\chi} \gamma^{\mu} \gamma_{5} \chi}{\Lambda^{2}} \sum_{i=1}^{3}\left[\bar{u}_{i} \gamma_{\mu}\left(c_{\chi U_{i}} P_{R}+c_{\chi Q_{i}} P_{L}\right) u_{i}+\bar{d}_{i} \gamma_{\mu}\left(c_{\chi D_{i}} P_{R}+c_{\chi Q_{i}} P_{L}\right) d_{i}\right]
$$

where all coefficients $c_{\chi U_{i}}, c_{\chi D_{i}}$, and $c_{\chi Q_{i}}$ are real numbers.

\section{Cosmological and astrophysical constraints}

We are now ready to put limits on the Wilson coefficients $c_{i}$. In this section, we consider various limits obtained from cosmological and astrophysical observations, while those obtainable from the LHC experiments will be discussed in the next section. For the real scalar DM $\phi$, twelve operators, viz. $\phi^{2} \bar{u}_{i} u_{i}, \phi^{2} \bar{u}_{i} i \gamma_{5} u_{i}, \phi^{2} \bar{d}_{i} d_{i}$, and $\phi^{2} \bar{d}_{i} i \gamma_{5} d_{i}$ are considered, where the index $i$ runs over the families. On the other hand, for the Majorana fermion DM $\chi$, the nine operators $\left(\bar{\chi} \gamma^{\mu} \gamma_{5} \chi\right)\left(\bar{u}_{i} \gamma_{\mu} P_{R} u_{i}\right)$, $\left(\bar{\chi} \gamma^{\mu} \gamma_{5} \chi\right)\left(\bar{d}_{i} \gamma_{\mu} P_{R} d_{i}\right)$, and $\left(\bar{\chi} \gamma^{\mu} \gamma_{5} \chi\right)\left(\bar{u}_{i} \gamma_{\mu} P_{L} u_{i}+\bar{d}_{i} \gamma_{\mu} P_{L} d_{i}\right)$ are of interest. As new physics would generally result in several operators in the effective low-energy theory, putting limits on individual operators in isolation should be regarded as only an indicative exercise. Keeping this fact in mind, we simply assume that the effective lagrangian has only one of the operators mentioned above and derive the corresponding bounds.

\subsection{Cosmological limits}

The thermal relic abundance of the DM [5] imposes an important constraint. A small annihilation cross section of the DM implies a larger resultant abundance, leading possibly to the over-closure of the universe. This immediately leads to a lower limit on the coefficient of the operator from the cross section. Indeed, a consonance with the WMAP results would lead to an even more restrictive bound. 
For the scalar DM $\phi$ having interactions $\left(v \phi^{2} / \sqrt{2} \Lambda^{2}\right)\left(c^{(R)} \bar{q} q+c^{(I)} \bar{q} i \gamma_{5} q\right)$, where $q$ represents a quark $\left(q=u_{i}, d_{i}\right)$ with $m_{q}$ being its mass, the annihilation cross section of the $\mathrm{DM} \phi$ (times relative velocity $v_{\text {rel }}$ ) is evaluated to be

$$
\left.\sigma v_{\mathrm{rel}}\right|_{\phi \phi} \simeq \frac{3 v^{2}}{2 \pi \Lambda^{4}}\left[\left(c^{(R)}\right)^{2}\left(1-m_{q}^{2} / m_{\phi}^{2}\right)^{3 / 2}+\left(c^{(I)}\right)^{2}\left(1-m_{q}^{2} / m_{\phi}^{2}\right)^{1 / 2}\right]+\mathcal{O}(\epsilon)
$$

where $\epsilon=\left(s-4 m_{\phi}^{2}\right) /\left(4 m_{\phi}^{2}\right)$. Using a semi-analytical formula for the DM abundance [6], we obtain a lower limit on the coefficient $\sqrt{c^{(R)}} / \Lambda$ or $\sqrt{c^{(I)}} / \Lambda$.

On the other hand, for the Majorana fermion DM $\chi$ governed by the interaction $\left(2 c / \Lambda^{2}\right)\left(\bar{\chi} \gamma_{\mu} \gamma_{5} \chi\right)\left(\bar{q} \gamma^{\mu} P_{R(L)} q\right)$, the annihilation cross section reads

$$
\left.\sigma v_{\mathrm{rel}}\right|_{\chi \chi} \simeq \frac{4 c^{2}}{\pi \Lambda^{4}}\left(4 m_{\chi}^{2} \epsilon+3 m_{q}^{2} / 2\right) \sqrt{1-m_{q}^{2} / m_{\chi}^{2}},
$$

where $\epsilon=\left(s-4 m_{\chi}^{2}\right) /\left(4 m_{\chi}^{2}\right)$ again. As in the case of the scalar DM, we obtain a lower limit on the coefficient $\sqrt{c} / \Lambda$ using the same semi-analytical formula.

\subsection{Astrophysical limit 1 (Direct detection)}

Direct detection experiments put a stringent limit on a Wilson coefficient when the corresponding operator contributes to the spin-independent cross section for the DM scattering off a nucleon. In this paper, we adopt the results of the XENON100 collaboration [2]. For the scalar DM $\phi$, as shown in eqn.(3), there are two types of interactions, namely scalar and pseudo-scalar. Since the pseudo-scalar interaction is not constrained, the operators of the scalar DM are limited only through the scalar interaction.

Using the formula discussed in Ref. [7], the spin-independent scattering cross section between the DM and a nucleon is given by the following formula when the DM couples to light quarks ( $u, d$, and $s$ quarks):

$$
\sigma_{\mathrm{SI}}=\frac{\left(c^{(R)}\right)^{2} v^{2} m_{N}^{4} f_{T q}^{2}}{2 \pi \Lambda^{4}\left(m_{\phi}+m_{N}\right)^{2} m_{q}^{2}}
$$

where $m_{N} \simeq 940 \mathrm{MeV}$ is the nucleon mass, while $f_{T q}$ is the parameter determined by the hadron matrix element $\langle N|\bar{q} q| N\rangle$. The values of this parameter for $u, d$, and $s$ quarks are $f_{T u} \simeq 0.028, f_{T d} \simeq 0.028$, and $f_{T s} \simeq 0.009$, respectively [8]4. On the

\footnotetext{
${ }^{4}$ These values, which are obtained by the lattice QCD calculations, are consistent with the ones extracted from the $\pi-N$ scattering data with the aid of the chiral perturbation theory 9 .
} 
other hand, when the DM couples to heavy quarks ( $c, b$, and $t$ quarks), the formula of the spin-independent scattering cross section changes to

$$
\sigma_{\mathrm{SI}}=\frac{\left(c^{(R)}\right)^{2} 2 v^{2} m_{N}^{4} f_{T G}^{2}}{729 \pi \Lambda^{4}\left(m_{\phi}+m_{N}\right)^{2} m_{q}^{2}} .
$$

The parameter $f_{T G}$ is determined by the hadron matrix element $\left\langle N\left|G_{\mu \nu}^{a} G^{a \mu \nu}\right| N\right\rangle$ and its value is evaluated to be $f_{T G} \simeq 0.9431$ through the trace anomaly relation, namely $f_{T u}+f_{T d}+f_{T s}+f_{T G}=1$. The essential reason why the scattering cross section depends on the gluon hadron matrix element is that the DM can scatter off a nucleon through an effective interaction with gluons inside the nucleon, a process mediated by a one-loop diagram involving the heavy quark 5 .

For a Majorana fermion, the charge radius vanishes identically, and all interactions with quarks involve the axial-vector current, namely $\bar{\chi} \gamma_{\mu} \gamma_{5} \chi$. Only the spatial components of the interaction survive at the non-relativistic limit, and the corresponding quark interaction become $6 \bar{q} \gamma^{i} \gamma_{5} q$. These operators, therefore, contribute only to the spin-dependent scattering cross section, but not to the spin-independent one. Since the limit on the former cross section from direct detection experiments turns out to be much weaker than that obtained from the LHC experiments, we do not consider this constraint.

\subsection{Astrophysical limit 2 (Indirect detection)}

Several products from DM annihilations in the galactic halo, for example, gammarays, positrons, electrons, anti-protons, and anti-deuterons, are utilized to detect the DM. Among these, one of the most reliable limits on the annihilation cross section of DM comes from the indirect detection experiment through gamma-ray observations. In particular, the observations from the galactic center and Milky Way satellites currently give the most stringent limit. In this article, we put a limit on each operator according to Ref. [10], in which a bound on the DM annihilation cross section obtained by the gamma-ray observation from the galactic center at the Fermi-LAT experiment is presented.

The differential gamma-ray flux in a direction $\psi$ originated in DM annihilations

\footnotetext{
${ }^{5}$ For light quarks, contributions to the scattering cross section from the loop-diagrams have a form different from that in eqn.(9) and are suppressed by the mass of light quarks.

${ }^{6}$ Note that $\left\langle N\left|\bar{q} \gamma^{i} q\right| N\right\rangle=0$ in the non-relativistic limit.
} 
at the region around the galactic center is given by

$$
\Phi(\psi)=\frac{1}{4 \pi} \frac{\sigma v_{\mathrm{rel}}}{2 m_{\mathrm{DM}}^{2}}\left[\frac{d N_{\gamma}}{d E}\right] \int_{l . o . s} d l \rho^{2}(l),
$$

where $\sigma$ is the annihilation cross section with $v_{\text {rel }}$ being the relative velocity between the two incident DM particles, each of mass $m_{\mathrm{DM}}$. The mass density of DM (the DM profile) at the position $l$ is denoted by $\rho(l)$, and the integration is performed along the observer's line-of-sight. Of the many profiles discussed in the literature, we use the "Cored $\left(R_{c}=1 \mathrm{kpc}\right)$ " DM profile as it gives the most conservative limit on the annihilation cross section. The fragmentation function $d N_{\gamma} / d E$, which is nothing but the gamma-ray spectrum produced per annihilation, has been estimated using the PYTHIA code [12] for each possible final state for the primary process, viz. $u \bar{u}$, $s \bar{s}, b \bar{b}, t \bar{t}$, etc.

Using the result of the Fermi-LAT experiment, model independent upper limits on the bin-integrated quantity $\left(\sigma v_{\text {rel }} / m_{\mathrm{DM}}^{2}\right) \times \int_{E_{i}}^{E_{f}} d E\left[d N_{\gamma} / d E\right]$ for various energy bins, $\left(E_{i}, E_{f}\right)=(0.3,1),(1,3),(3,10)$, and $(10,100)$ in $\mathrm{GeV}$ unit, are presented in Ref. [10]. The upper limit on the annihilation cross section, $\sigma v_{\text {rel }}$, is therefore obtained when the DM mass and the fragmentation function are given. As the DM velocity at the present universe is three orders of magnitude smaller than the speed of light, the product $\left(\sigma v_{\text {rel }}\right)$ is dominated by the $s$-wave component. For the scalar $\mathrm{DM}$, this is given by

$$
\sigma v_{\mathrm{rel}} \simeq \frac{3 v^{2}}{2 \pi \Lambda^{4}}\left[\left(c^{(R)}\right)^{2}\left(1-m_{q}^{2} / m_{\phi}^{2}\right)^{3 / 2}+\left(c^{(I)}\right)^{2}\left(1-m_{q}^{2} / m_{\phi}^{2}\right)^{1 / 2}\right]
$$

where the interaction of the DM is assumed to be $\left(v \phi^{2} / \sqrt{2} \Lambda^{2}\right)\left(c^{(R)} \bar{q} q+c^{(I)} \bar{q} i \gamma_{5} q\right)$. On the other hand, for the Majorana fermion DM $\chi$, the s-wave component of the annihilation cross section is given by

$$
\sigma v_{\mathrm{rel}} \simeq \frac{6 c^{2} m_{q}^{2}}{\pi \Lambda^{4}} \sqrt{1-m_{q}^{2} / m_{\chi}^{2}}
$$

where the interaction Lagrangian is $\left(2 c / \Lambda^{2}\right)\left(\bar{\chi} \gamma_{\mu} \gamma_{5} \chi\right)\left(\bar{q} \gamma^{\mu} P_{R(L)} q\right)$. Since the annihilation cross section is proportional to the fermion mass squared (on account of the helicity suppression), only the interactions involving the second and third generation quarks are constrained by the indirect detection experiment.

In this section, we have discussed the method to put limits on the DM operators using cosmological and astrophysical observations. Obtained limits on each operator 
are summarized in Fig 3 for the real scalar DM and Fig, 4 for the Majorana fermion DM in section 5, where limits from cosmology, direct detection, and indirect detection experiments are shown as brown, green, and blue lines in both figures. Physical interpretation of the results are discussed in section 5 in comparison with the limits obtained from the LHC experiment.

\section{Constraints from the LHC experiment}

We first discuss possible signals of DM at the LHC experiment. DM particles are pairproduced from quark-quark interactions through the higher dimensional operators discussed in section 2. However, exclusive pair production does not result in a visible object to tag on to and, hence, cannot be detected by the experiment. We, thus, need to pair-produce DM particles in association with jets or photons. In this case, the DM pair recoils against the visible particle(s) and produces missing transverse energy $\left(\mathbb{E}_{T}\right)$. The generic signature of DM production at the LHC experiment is thus $\mathbb{E}_{T}$ plus a few number of jets or photons.

In the context of LHC searches, we can divide the DM effective operators into three categories, i.e., the operators containing light quarks, bottom quarks and top quarks respectively. In the first case, we can pair-produce DM particles with gluon/quark radiations from initial quark/gluon legs and thus observe the signal in the mono-jet plus $\mathbb{E}_{T}$ channel. In the second case, we need two bottom quarks in the initial state that may come from the gluon splitting 7 . This means, we may produce DM pair alongwith two b-jets. The DM interaction with top quarks is very different from the other cases. Here we need to produce two DM particles in association with two top quarks and the signal looks like top pair plus $\mathbb{E}_{T}$. This final state is particularly interesting as it comes from different beyond the SM processes.

From the discussions above, it is clear that the signals will comprise events with $\mathbb{E}_{T}$ plus a few of SM particles with the hope that the shape of the $\mathbb{E}_{T}$ distribution may be used to distinguish these from the SM backgrounds. However, unlike the Rparity conserving SUSY, $\mathbb{E}_{T}$ comes from the recoil of jets against DM particles and thus produces featureless rapidly decreasing distribution for DM coupled to light and bottom quarks. In case of top quark-DM effective operators, the situation is slightly different as $\mathbb{E}_{T}$ can also come from leptonic decays of $W$ bosons. It is therefore

\footnotetext{
${ }^{7}$ Note that a gauge-invariant calculation would also need to included diagrams such as $g b \rightarrow b \phi \phi$ with the initial $b$ emanating from gluon spitting.
} 


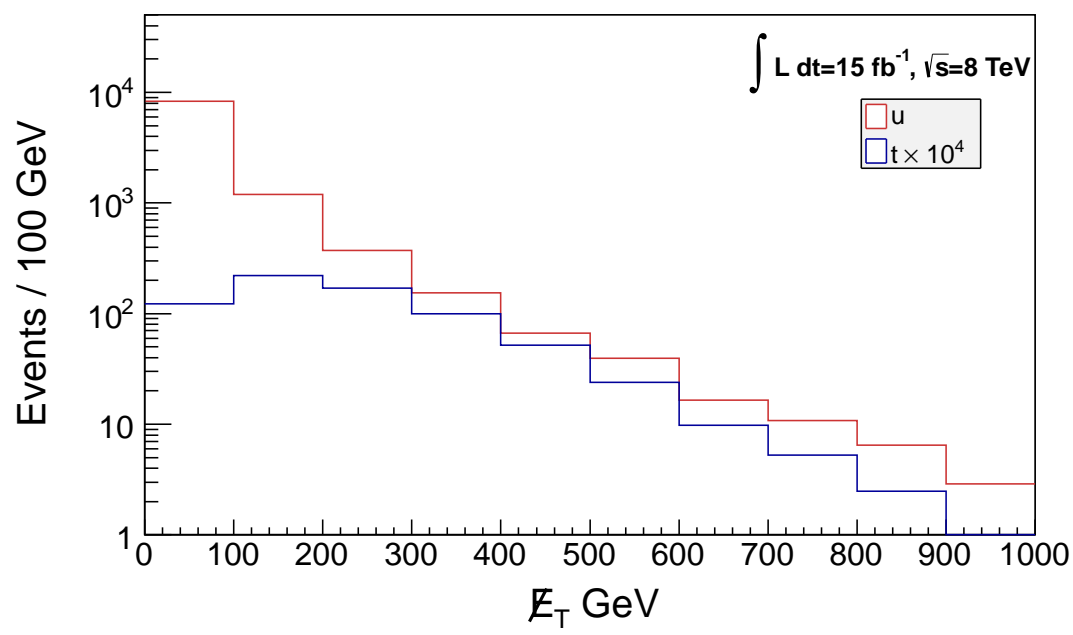

Figure 1: $\mathbb{E}_{T}$ distributions for the two operators described in the text at $8 \mathrm{TeV}$ run.

important to compare the $\mathbb{E}_{T}$ distributions for these two cases. The $\mathbb{E}_{T}$ distribution becomes harder with increasing the DM mass although the overall production cross section decreases with the mass.

For illustrative purposes, consider the two operators $\left(2 \bar{\chi} \gamma^{\mu} \gamma_{5} \chi\right)\left(\bar{u} \gamma_{\mu} P_{R} u\right) / \Lambda^{2}$ and $\left(2 \bar{\chi} \gamma^{\mu} \gamma_{5} \chi\right)\left(\bar{t} \gamma_{\mu} P_{R} t\right) / \Lambda^{2}$ assuming $m_{\chi}=100 \mathrm{GeV}$ and $\Lambda=1 \mathrm{TeV}$. The corresponding $\mathbb{E}_{T}$ distributions are shown in Fig 1 for the $8 \mathrm{TeV}$ run with $15 \mathrm{fb}^{-1}$ integrated luminosity. It is immediately apparent that the $\mathbb{E}_{T}$ distribution for the top-quark case is slightly harder compared to the light quark case. However, with the cross section being much smaller, it is not possible to probe a DM that couples primarily to top quarks (and with a strength comparable to the electroweak interactions) from the current $(8 \mathrm{TeV})$ run and we have to consider high luminosity LHC run $\left(100 \mathrm{fb}^{-1}\right)$ with $\sqrt{s}=14 \mathrm{TeV}$.

\subsection{Model independent upper limits}

We now discuss upper limits on the cross section times acceptances for aforementioned interactions. We consider three specific channels, namely mono jet $+\mathbb{E}_{T}$, mono b-jet $+\mathbb{E}_{T}$ and top quark(s) $+\mathbb{E}_{T}$ searches. In order to estimate the prospects, we perform Monte-Carlo (MC) simulations. Madgraph5 [11] is utilized to generate parton level events and the events are interfaced to PYTHIA6.420 [12] to deal with showering and hadronization. For detector simulation, we utilize Delphes2.0.2 [13], 
which takes into account effects of mis-measurements in a $\mathrm{MC}$ way, and resolution parameters are chosen based on the CMS detector performance given in Ref. [14].

\subsubsection{Mono jet $+\mathbb{E}_{T}$}

The upper limit on the non-standard model contribution to the mono jet $+\mathbb{E}_{T}$ channel have already been reported in Refs. [14, 15, 16] for the $7 \mathrm{TeV}$ run. In this paper, we take the results of Ref. [15] and set the constraints. We also estimate the prospect for $8 \mathrm{TeV}$ run by the $\mathrm{MC}$ simulation imitating the search discussed in the reference. The basic selections used in the analysis are the following:

1. Event must have a missing transverse energy $\mathbb{E}_{T}>200 \mathrm{GeV}$.

2. The leading jet must have $p_{T}>110 \mathrm{GeV}$ and $|\eta|<2.4$.

3. The number of jets with $p_{T}>30 \mathrm{GeV}$ needs to be smaller than 3 .

4. There should be no isolated leptons or tracks with $p_{T}>10 \mathrm{GeV}$.

5. The second jet must have $p_{T}<30 \mathrm{GeV}$ or the difference of the azimuthal angle between the leading and the second jets should be smaller than 2.5.

Here, $p_{T}$ and $\eta$ denote the transverse momentum and pseudo-rapidity respectively. In Ref. [15], the upper limit on the number of the non-standard model events has been obtained, when an additional cut of $\mathbb{E}_{T}>250,300,350$, or $400 \mathrm{GeV}$ is applied. We express the results in terms of the cross section times the acceptance $(\sigma \times \mathcal{A})$, which are summarized in the table shown below;

\begin{tabular}{c|cccc} 
Cut on $\notin_{T}(\mathrm{GeV})$ & 250 & 300 & 350 & 400 \\
\hline$\sigma \times \mathcal{A}(\mathrm{fb})$ & 120 & 73.6 & 31.6 & 19.0 \\
\hline
\end{tabular}

In order to estimate the prospect with the $8 \mathrm{TeV}$ run as accurately as possible, we first perform the $\mathrm{MC}$ simulation for a $7 \mathrm{TeV}$ run and compare the background numbers with those of Ref. [15] and obtain normalization factors required to adjust the difference. The same normalization factors are used to calculate the backgrounds for the $8 \mathrm{TeV}$ run 8 . Note that the cross section obtained by MadGraph and PYTHIA is only at LO, and one needs a $\mathrm{K}$ factor to obtain the correct normalization. Instead using the $\mathrm{K}$ factor, we compare our simulation to the distribution in the reference. As is shown in the reference, $W \rightarrow l \nu+$ jets, $Z \rightarrow \nu \nu+$ jets and $t \bar{t}+$ jets are the

\footnotetext{
${ }^{8}$ This approximation is expected to be quite an accurate one as the modest increase in the operating energy does not change the features of the distributions to an appreciable degree.
} 


\begin{tabular}{c|ccc} 
& $W \rightarrow l \nu+$ jets & $Z \rightarrow \nu \nu+$ jets & $t \bar{t}+$ jets \\
\hline Cut 1-3 & 176369 & 62634 & 4756 \\
Cut 4 & 157616 & 58216 & 4016 \\
Cut 5 & 39084 & 56597 & 625 \\
$\mathbb{E}_{T}>250 \mathrm{GeV}$ & 12915 & 21986 & 193 \\
$\mathbb{E}_{T}>300 \mathrm{GeV}$ & 4950 & 9588 & 59 \\
$\mathbb{E}_{T}>350 \mathrm{GeV}$ & 2065 & 4674 & 28 \\
$\mathbb{E}_{T}>400 \mathrm{GeV}$ & 928 & 2337 & 20 \\
$\mathbb{E}_{T}>450 \mathrm{GeV}$ & 473 & 1275 & 8 \\
$\mathbb{E}_{T}>500 \mathrm{GeV}$ & 223 & 754 & 7 \\
\hline
\end{tabular}

Table 1: Cut flow table for the SM backgrounds at the mono jet $+\mathbb{E}_{T}$ channel. The center of mass energy of $8 \mathrm{TeV}$ and the integrated luminosity of $15 \mathrm{fb}^{-1}$ are assumed.

dominant backgrounds, where $l$ denotes a charged lepton. Other possible sources such as QCD, single top, etc. have negligible contributions. The cut flow for the standard model events is shown in Table 1 assuming an integrated luminosity of 15 $\mathrm{fb}^{-1}$.

We have calculated the expected 95\% C.L. upper limit on the non-standard model cross section times the acceptance, which is summarized in the table below. Here, we have assumed that the systematic uncertainty associated with the estimation of the SM background events is $10 \%$, which is same as the one in Ref. [15].

\begin{tabular}{c|cccccc} 
Cut on $\not_{T}(\mathrm{GeV})$ & 250 & 300 & 350 & 400 & 450 & 500 \\
\hline$\sigma \times \mathcal{A}(\mathrm{fb})$ & 459 & 191 & 89.1 & 43.6 & 23.7 & 13.7 \\
\hline
\end{tabular}

\subsubsection{Mono b-jet $+\mathbb{E}_{T}$}

The mono b-jet $+\mathbb{E}_{T}$ channel is expected to be useful to search for a DM that couples mainly to bottom quarks. In addition to the selection cuts used in the previous subsection (the cuts $1-5$ ), we also require

6. The leading jet is b-tagged.

For the b-tagging method, we assume the following properties:

- The b-tagging efficiency is 0.6 for real b jets.

- The mis-tagging rate for light jet is 0.004 . 


\begin{tabular}{c|ccc} 
& $W \rightarrow l \nu+$ jets & $Z \rightarrow \nu \nu+$ jets & $t \bar{t}+$ jets \\
\hline $\mathbb{E}_{T}>350 \mathrm{GeV}$ & 33 & 111 & 23 \\
\hline
\end{tabular}

Table 2: Expected number of the SM events in the mono b-jet $+\mathbb{E}_{T}$ channel. A center of mass energy of $8 \mathrm{TeV}$ and an integrated luminosity of $15 \mathrm{fb}^{-1}$ are assumed.

- The mis-tagging rate for c-jet is 0.1 .

These tagging efficiencies can be achieved in the CSVM tagger calibrated in Ref. [17. With the b-jet being the leading one, a large $\mathbb{E}_{T}$ would, typically, require a large $p_{T}$ for the b-jets. This, though, would lower the efficiency of b-tagging. from the value quoted above. Hence, we do not raise the $\mathbb{E}_{T}$-cut to levels as high as those for the case of the mono jet $+\mathbb{E}_{T}$ channel, and just impose a moderate requirement of $\mathbb{E}_{T}>350 \mathrm{GeV}$.

The estimation of the number of the SM events after the cuts 1-6 and $\mathbb{E}_{T}>$ $350 \mathrm{GeV}$ is shown in Table 2 for $8 \mathrm{TeV}$ run assuming $15 \mathrm{fb}^{-1}$ data. Here, "jets" also include those originating in heavy flavors. Since the mono b-jet $+\mathbb{E}_{T}$ channel is almost similar to the mono jet $+\mathbb{E}_{T}$ channel, the dominant backgrounds in the previous subsection are also the dominant ones here. We use the same normalization factor as the one in the mono jet $+\mathbb{E}_{T}$ channel to estimate the number of the events.

The expected 95\% C.L. upper limit on the cross section times the acceptance for the non-SM event is estimated to be $\mathbf{2 . 9} \mathbf{~ f b}$. Here, we have again assumed that the systematic uncertainty in the estimation of the SM events is $10 \%$. One may expect that the use of tighter b-tagging condition would efficiently reduce the $W$ and $Z$ backgrounds. However, it turns out that half of the remaining $W$ and $Z$ events include the real b-jets with the b-tagging properties we adopt. Therefore, tightening the b-tagging condition does not improve the result of our analysis.

\subsubsection{Top quark(s) $+\mathbb{E}_{T}$}

The case of the DM coupling primarily to the top quark needs separate treatment. At the LHC, such a DM can only be pair-produced in association with two top quarks, and the production cross section is highly suppressed in comparison to the case of light quarks. To compensate for this, we concentrate on the prospects for the $14 \mathrm{TeV}$ run, rather than the the 7(8) $\mathrm{TeV}$ ones.

The top quark decays promptly to a hard b-jet and an on-shell $W$. If the $W$, 
in turn, decays into a lepton-neutrino pair, the last-mentioned would contribute to missing momentum. In other words, both the semileptonic and dileptonic decay channels for the $t \bar{t}$ pair are associated with an inherent $\mathbb{E}_{T}$, and recognizing the additional missing momentum due to the DM pair would be a nontrivial process. Indeed for such channels (i.e., semileptonic or dileptonic decays), the SM $t \bar{t}$ production itself proves to be an almost insurmountable source of background to the DM signal. In order to suppress this, we consider instead the pure hadronic mode (even with all the associated complications). We, therefore, impose the following basic selection cuts:

1. There is no isolated light lepton $\left(e^{ \pm}, \mu^{ \pm}\right)$with $p_{T}>20 \mathrm{GeV}$.

2. There are at least four jets with $p_{T}>100,80,50,50 \mathrm{GeV}$ (sequentially) and one of them has to be b-tagged.

3. The azimuthal angles between the missing transverse momentum and the leading four jets are larger than 0.2 .

4. $M_{\text {eff }}>1000 \mathrm{GeV}$ and $\mathbb{E}_{T} / M_{\text {eff }}>0.3$.

Here, $M_{\text {eff }}$ is the scalar sum of the transverse momenta of the leading 4 jets and $\mathbb{E}_{T}$.

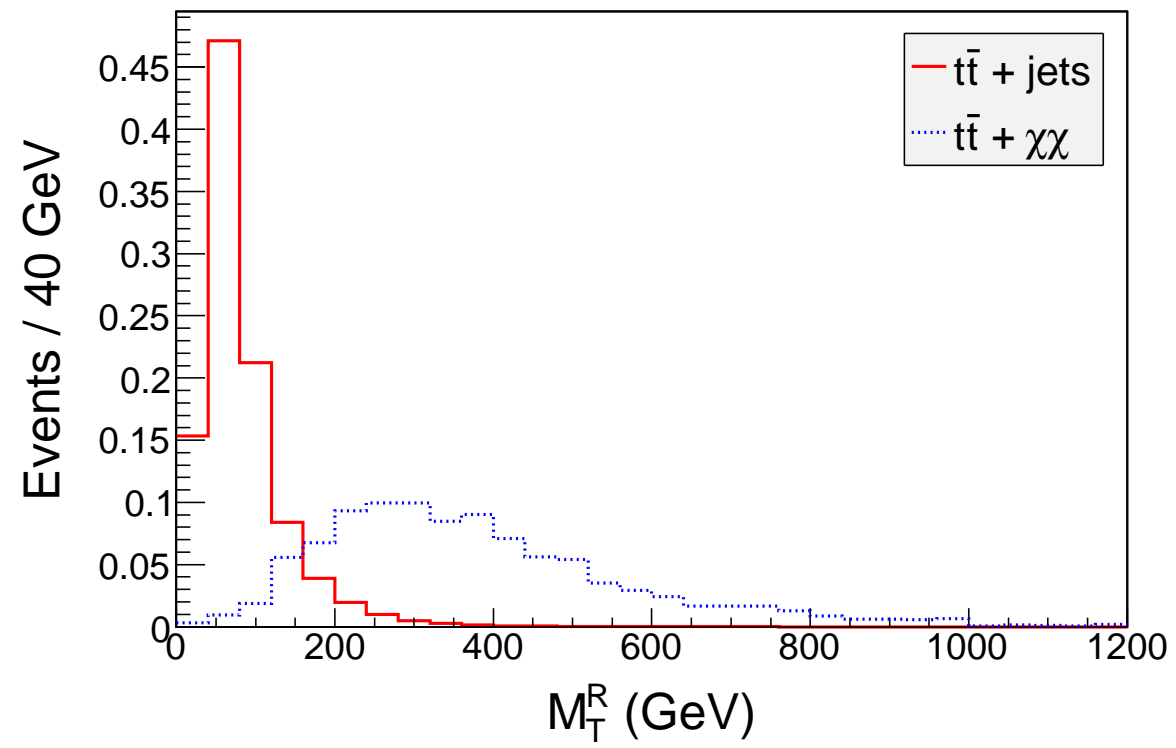

Figure 2: Normalized distribution of the $M_{T}^{R}$ variable for the SM background $(t \bar{t})$ and for DM effective operator $\left(2 \bar{\chi} \gamma^{\mu} \gamma_{5} \chi\right)\left(\bar{t} \gamma_{\mu} P_{R} t\right) / \Lambda^{2}$ events after imposing the selection cut $1-3$. Center of mass energy of the LHC experiment is set to be $14 \mathrm{TeV}$. 


\begin{tabular}{c|c|c|c} 
& $t \bar{t}+$ jets & $Z(\rightarrow \nu \nu)+$ jets & $W(\rightarrow l \nu)+$ jets \\
\hline Cut $1-4$ & 5746 & 258 & 176 \\
$M_{T}^{R}>300 \mathrm{GeV}$ & 5723 & 255 & 176 \\
$M_{T}^{R}>400 \mathrm{GeV}$ & 4753 & 239 & 174 \\
$M_{T}^{R}>500 \mathrm{GeV}$ & 1934 & 200 & 141 \\
$M_{T}^{R}>600 \mathrm{GeV}$ & 659 & 143 & 95 \\
$M_{T}^{R}>700 \mathrm{GeV}$ & 239 & 85 & 50 \\
$M_{T}^{R}>800 \mathrm{GeV}$ & 94 & 47 & 19 \\
$M_{T}^{R}>900 \mathrm{GeV}$ & 21 & 21 & 14 \\
\hline
\end{tabular}

Table 3: Expected cut flow for the SM backgrounds in the top $+\mathbb{E}_{T}$ channel. The center of mass energy of $14 \mathrm{TeV}$ and the integrated luminosity of $100 \mathrm{fb}^{-1}$ are assumed.

The top quark(s) $+\mathbb{E}_{T}$ channel resembles the supersymmetry search with multijet $+\mathbb{E}_{T}$ [18]. The dominant backgrounds accrue from $W+$ jets (with $W \rightarrow l \nu$ ), $Z+$ jets (with $Z \rightarrow \nu \nu$ ) and $t \bar{t}+$ jets. Interestingly, we find that the $M_{T}^{R}$ variable [19] is useful in separating the signal events from the SM $t \bar{t}+$ jets events. In order to define the variable, we first define two mega jets. If $n$ jets are present in the event, $2^{n-1}$ different combinations are possible in such a reconstruction. Of these, we choose the one which minimizes the sum of the squares of the two mega jet masses. From the four momentum of the two mega jets $p^{j 1}, p^{j 2}$ and the transverse missing momentum $\vec{E}_{T}$, the quantity $M_{T}^{R}$ is defined by

$$
M_{T}^{R}=\sqrt{\frac{\mathbb{E}_{T}\left(p_{T}^{j 1}+p_{T}^{j 2}\right)-\overrightarrow{\mathbb{E}}_{T} \cdot\left(\vec{p}_{T}^{j 1}+\vec{p}_{T}^{j 2}\right)}{2}} .
$$

For the SM $t \bar{t}$ events, the distribution falls sharply for $M_{T}^{R} \gtrsim m_{t}$, while that for the signal is expected to be broader. We show the $M_{T}^{R}$ distributions for the SM $t \bar{t}$ and for the DM effective operator $\left(2 \bar{\chi} \gamma^{\mu} \gamma_{5} \chi\right)\left(\bar{t} \gamma_{\mu} P_{R} t\right) / \Lambda^{2}$ in Fig. 2 assuming $m_{\chi}=100 \mathrm{GeV}$ and $\Lambda=1 \mathrm{TeV}$ after imposing the selection cuts $1-3$. Based on this distribution, we impose additional cuts $M_{T}^{R}>300,400,500,600,700,800$ and $900 \mathrm{GeV}$. The cut flow for the SM backgrounds, namely $t \bar{t}+$ jets, $W+$ jets, and $Z+$ jets backgrounds are shown in Table 3 .

As expected, the $t \bar{t}+$ jets background reduces drastically with stronger $M_{T}^{R}$ cuts, whereas the $Z$ and $W$ backgrounds are not reduced significantly. However, given that the latter are inherently smaller, this is not worrisome. Here, the number of the $t \bar{t}$ events is normalized assuming the total cross section of $920 \mathrm{pb}$ [20]. The numbers 
of the $W \rightarrow l \nu+$ jets and $Z \rightarrow \nu \nu+$ jets events are normalized with the matched cross section obtained from the MadGraph-PYTHIA package. We have calculated the expected $95 \%$ C.L. upper limit on the non-standard model cross section times the acceptance, which is shown in the table below. Here, the systematic uncertainty in the estimation of the standard model events was assumed to be $10 \%$, which is typical of supersymmetry searches with the hadronic mode.

\begin{tabular}{c|ccccccc} 
Cut on $M_{T}^{R}(\mathrm{GeV})$ & 300 & 400 & 500 & 600 & 700 & 800 & 900 \\
\hline$\sigma \times \mathcal{A}(\mathrm{fb})$ & 12.2 & 10.2 & 4.57 & 1.87 & 0.839 & 0.418 & 0.208 \\
\hline
\end{tabular}

In this paper, only the result for the hadronic mode has been shown. On the other hand, we also have estimated the prospects using the one (semileptonic) and two lepton (leptonic) modes. For the leptonic mode, we use the $M_{T 2}$ variable constructed from $\mathbb{E}_{T}$ and the transverse momentum of two leptons. For the semi-leptonic mode, the $M_{T}$ variable constructed from $\mathbb{E}_{T}$ and the transverse momentum of a lepton are used to reduce the background. These methods are discussed in Ref. [21] in the context of scalar top searches. We find, however, that the corresponding constraints are weaker than those obtained with the hadronic mode.

We are now ready to discuss the imposition of constraints on each operator from the LHC experiment. For this purpose, we implement the interactions described in section 2 in Madgraph5 with the aid of Feynrules [22] and perform MC simulations for three channels as discussed above.

In order to put constraints on the operators involving light quarks (up, down, charm and strange quarks), we perform the mono jet $+\mathbb{E}_{T}$ analysis. We find the optimized cut on $\mathbb{E}_{T}$ by maximizing the ratio of the efficiency for accepting the signal to the upper bound on the cross section times the acceptance for the non-standard model events. As a reference point, we choose the DM mass of $100 \mathrm{GeV}$ and the interaction with up quark 9 The results are summarized in Table 4 . As expected, a stronger cut on $\mathbb{E}_{T}$ gives more severe bound on the interactions for the Majorana fermion DM. It is possible that a cut on $\mathbb{E}_{T}$ even stronger than those discussed in section 4.1.1 would result in a more severe bound. However, such strong cuts typically lead to larger systematic errors, and, hence, we do not consider this avenue. The current and future expected constraints on each operator are shown in the first and the second columns of Fig 3 and 4 in the next section.

\footnotetext{
${ }^{9}$ The optimized values do not change by more than $50 \mathrm{GeV}$ even if we choose other reference
} 


\begin{tabular}{c|cc} 
& Real scalar & Majorana fermion \\
\hline $7 \mathrm{TeV}$ & $350 \mathrm{GeV}$ & $400 \mathrm{GeV}$ \\
$8 \mathrm{TeV}$ & $500 \mathrm{GeV}$ & $500 \mathrm{GeV}$ \\
\hline
\end{tabular}

Table 4: Optimized cut on $\mathbb{E}_{T}$ for the real scalar and the Majorana fermion DMs.

In order to put constraints on the operators involving bottom and top quarks, we perform the mono b-jet $+\mathbb{E}_{T}$ and the top quarks(s) $+\mathbb{E}_{T}$ analysis, respectively. For the top quarks(s) $+\mathbb{E}_{T}$ analysis, the optimized cut on $M_{T}^{R}$ turns out to be 900 $\mathrm{GeV}$ for both the real scalar and the Majorana fermion DMs. The future expected constraints on the operators are shown in the third columns of Figs. 3 and 4 ,

\section{Results}

We have already explained in the preceding two sections that the limits come mainly from WMAP, Fermi-LAT, XENON100 and LHC experiments. Since the structures of the operators are very different for the real scalar and the Majorana fermion DMs, we discuss the results of these two classes of interactions separately. In all cases, we vary the DM mass from $10 \mathrm{GeV}$ to $1 \mathrm{TeV}$ and calculate the limits and future prospects in terms of the only relevant combination of the cut-off scale $(\Lambda)$ and the coupling $(c)$, namely $\sqrt{c} / \Lambda$.

\subsection{Scalar DM}

All the (expected) limits for the real scalar DM interactions are summarized in Fig.3, where constraints from cosmology, direct detection, and indirect detection experiments are shown as brown, green, and blue lines, while the current limit and future prospect from the LHC experiment are shown as magenta and pink lines, respectively. It can be seen that the limits obtained from the indirect detection are much stronger than those from the LHC experiment except for the operators $\phi^{2} \bar{t} t$ and $\phi^{2} \bar{t} \gamma^{5} t$. This is because the production cross section at the LHC experiment is small for the scalar DM, while its annihilation cross section is large as there is neither helicity nor p-wave suppression.

The limit from the direct detection experiment is also very strong for the scalar points. 
interactions, since they contribute to the spin-independent scattering cross section. The limit on the operator $\phi^{2} \bar{c} c$ is stronger than that on $\phi^{2} \bar{s} s$. This is so as the hadronic matrix element $\langle N|\bar{s} s| N\rangle$ is small $\left(f_{T_{s}} \simeq 0.009\right)$, while the interaction with $c$ quark is expressed by the interaction with gluons at low energy and the corresponding matrix element $\left\langle N\left|G_{\mu \nu}^{a} G^{a \mu \nu}\right| N\right\rangle$ is large $\left(f_{T_{G}} \simeq 0.9431\right)$.

It is worth noting that the lines obtained from the cosmological limit are almost independent of the DM mass except for the top quark mass threshold for the operators $\phi^{2} \bar{t} t$ and $\phi^{2} \bar{t} \gamma^{5} t$. This is so because the annihilation cross section of the scalar DM is controlled by the dimension-5 operator after the electroweak symmetry breaking, and the cross section depends very weakly on the DM mass.

It is also clear that the scalar interactions with $u$ and $d$ quarks are not favored from current data, whereas the interaction with top quark is the most difficult one to constrain. Even with $100 \mathrm{fb}^{-1}$ data at $14 \mathrm{TeV}$ run, it would be challenging to cover most of the parameter space consistent with WMAP data.

\subsection{Fermion DM}

For the Majorana fermion DM, all results are summarized in Fig, 4 , where the (expected) limits are shown in the same way as for the scalar DM case. A remarkable point is that the limit from the indirect detection experiment is weak due to the helicity suppression. It is to be noted that the limit from the direct detection experiment is also very weak (and not shown in the figure), because the operators do not contribute to the spin-independent scattering cross section. It is therefore important to investigate the experimental way to search for the Majorana fermion DM at the LHC experiment.

The mono jet $+\mathbb{E}_{T}$ search gives strong limits on the DM interactions with light quarks (up, down, charm and strange quarks) in comparison with the limits obtained from the indirect detection experiment in the most of the parameter space, and so will the mono b-jet $+\mathbb{E}_{T}$ search on the interaction with bottom quark. Unfortunately, the limit from the $8 \mathrm{TeV}$ run will not be much stronger than the one obtained in the 7 $\mathrm{TeV}$ run despite the increase in signal cross section, as the number of SM background events increases as well. On the other hand, the top quark(s) $+\mathbb{E}_{T}$ search will give comparable limits to those from the indirect detection for the interaction with top quark, which is in sharp contrast to the case of the real scalar DM. 


\section{Summary}

We systematically investigate DM interactions with various quarks in the framework of effective operators. We consider the DM to be a singlet scalar or a Majorana fermion and thoroughly study bounds and future prospects of the DM discoveries from different astrophysical, cosmological and collider experiments. We have shown that the mono b-jet $+\mathbb{E}_{T}$, and the top quark(s) $+\mathbb{E}_{T}$ channels along with the mono jet $+\left(\mathbb{E}_{T}\right)$ channel could be very important to search for the DM interacting with quarks. Here, we derive model-independent upper limits on the cross section times acceptance for these three channels mentioned above at $7 \mathrm{TeV}$ ( 8 or $14 \mathrm{TeV}$ ) run of the LHC experiment. It is to be noted that the model independent bounds derived in our paper can also be used to constrain other DM or new physics models with similar type of signatures.

For the scalar DM with spin-independent interactions, the direct detection experiment supersedes the LHC bound because of smallness of the production cross section at the LHC experiment. Irrespective of whether it is spin-independent interaction or not, the indirect detection limit from Fermi-LAT data on the scalar DM is found to be stronger than the LHC bound. However, in case of the Majorana DM interactions considered in our work, the LHC experiment plays very important role for detection or exclusion of such possibility as the spin-independent cross section is zero. Possibility of detecting the top quark interaction with the DM is unfortunately not so promising at the LHC experiment even with $100 \mathrm{fb}^{-1}$ data at $14 \mathrm{TeV}$ run.

\section{Acknowledgments}

This work is supported by the Grant-in-Aid for Scientific research from the Ministry of Education, Science, Sports, and Culture (MEXT), Japan (Nos. 22244021, 23740169 for S.M., \& Nos. 22540300, 23104006 for M.M.N.), and also by the World Premier International Research Center Initiative (WPI Initiative), MEXT, Japan. DC thanks the Department of Science and Technology, India for assistance under the project DST-SR/S2/HEP-043/2009 and acknowledges partial support from the European Union FP7 ITN INVISIBLES (Marie Curie Actions, PITN-GA-2011-289442). The work of K.H. is supported by JSPS Research Fellowships for Young Scientists. 


\section{References}

[1] G. Bertone, (ed.), Cambridge, UK: Univ. Pr. (2010) 738 p.

[2] E. Aprile et al. [XENON100 Collaboration], arXiv:1207.5988 [astro-ph.CO].

[3] O. Adriani et al. [PAMELA Collaboration], Phys. Rev. Lett. 105, 121101 (2010);

M. Ackermann et al. [Fermi-LAT Collaboration], Phys. Rev. Lett. 107, 241302 (2011).

[4] Q. -H. Cao, C. -R. Chen, C. S. Li and H. Zhang, JHEP 1108, 018 (2011);

M. Beltran, D. Hooper, E. W. Kolb, Z. A. C. Krusberg and T. M. P. Tait, JHEP 1009, 037 (2010); J. Goodman, M. Ibe, A. Rajaraman, W. Shepherd, T. M. P. Tait and H. -B. Yu, Phys. Lett. B 695, 185 (2011); S. Kanemura, S. Matsumoto, T. Nabeshima and N. Okada, Phys. Rev. D 82, 055026 (2010);

J. Goodman, M. Ibe, A. Rajaraman, W. Shepherd, T. M. P. Tait and H. B. Yu, Phys. Rev. D 82, 116010 (2010); K. Cheung, K. Mawatari, E. Senaha, P. -Y. Tseng and T. -C. Yuan, JHEP 1010, 081 (2010); J. -M. Zheng, Z. -H. Yu, J. -W. Shao, X. -J. Bi, Z. Li and H. -H. Zhang, Nucl. Phys. B 854, 350 (2012); P. J. Fox, R. Harnik, J. Kopp and Y. Tsai, Phys. Rev. D 85, 056011 (2012); Z. H. Yu, J. -M. Zheng, X. -J. Bi, Z. Li, D. -X. Yao and H. -H. Zhang, Nucl. Phys. B 860, 115 (2012); K. Cheung, P. -Y. Tseng, Y. -L. S. Tsai and T. -C. Yuan, JCAP 1205, 001 (2012); P. J. Fox, R. Harnik, R. Primulando and C. -T. Yu, arXiv:1203.1662 [hep-ph]; Y. Bai and T. M. P. Tait, arXiv:1208.4361 [hep-ph]; U. Haisch, F. Kahlhoefer and J. Unwin, arXiv:1208.4605 [hep-ph].

[5] E. Komatsu et al. [WMAP Collaboration], Astrophys. J. Suppl. 192, 18 (2011) arXiv:1001.4538 [astro-ph.CO]].

[6] P. Gondolo and G. Gelmini, Nucl. Phys. B 360, 145 (1991).

[7] M. Drees and M. Nojiri, Phys. Rev. D 48, 3483 (1993); G. Jungman, M. Kamionkowski and K. Griest, Phys. Rept. 267, 195 (1996).

[8] H. Ohki et al. [JLQCD Collaboration], arXiv:1208.4185 [hep-lat].

[9] J. M. Alarcon, J. Martin Camalich and J. A. Oller, Phys. Rev. D 85, 051503 (2012); J. M. Alarcon, L. S. Geng, J. M. Camalich and J. A. Oller, arXiv:1209.2870 [hep-ph].

[10] D. Hooper, C. Kelso and F. S. Queiroz, arXiv:1209.3015 [astro-ph.HE].

[11] J. Alwall, M. Herquet, F. Maltoni, O. Mattelaer and T. Stelzer, JHEP 1106, 128 (2011). 
[12] T. Sjostrand, S. Mrenna and P. Z. Skands, JHEP 0605, 026 (2006).

[13] S. Ovyn, X. Rouby and V. Lemaitre, arXiv:0903.2225 [hep-ph].

[14] S. Chatrchyan et al. [CMS Collaboration], Phys. Rev. Lett. 107, 201804 (2011).

[15] S. Chatrchyan et al. [CMS Collaboration], arXiv:1206.5663 [hep-ex].

[16] [ALTAS Collaboration], ATLAS-CONF-2012-084.

[17] [CMS Collaboration], CMS-BTV-11-004.

[18] G. Aad et al. [ATLAS Collaboration], arXiv:0901.0512 [hep-ex].

[19] C. Rogan, arXiv:1006.2727 [hep-ph]; S. Chatrchyan et al. [CMS Collaboration], Phys. Rev. D 85, 012004 (2012); [CMS Collaboration], CMS-SUS-11-008.

[20] S. Moch and P. Uwer, Phys. Rev. D 78, 034003 (2008).

[21] T. Plehn, M. Spannowsky and M. Takeuchi, JHEP 1208, 091 (2012).

[22] N. D. Christensen and C. Duhr, Comput. Phys. Commun. 180, 1614 (2009). 


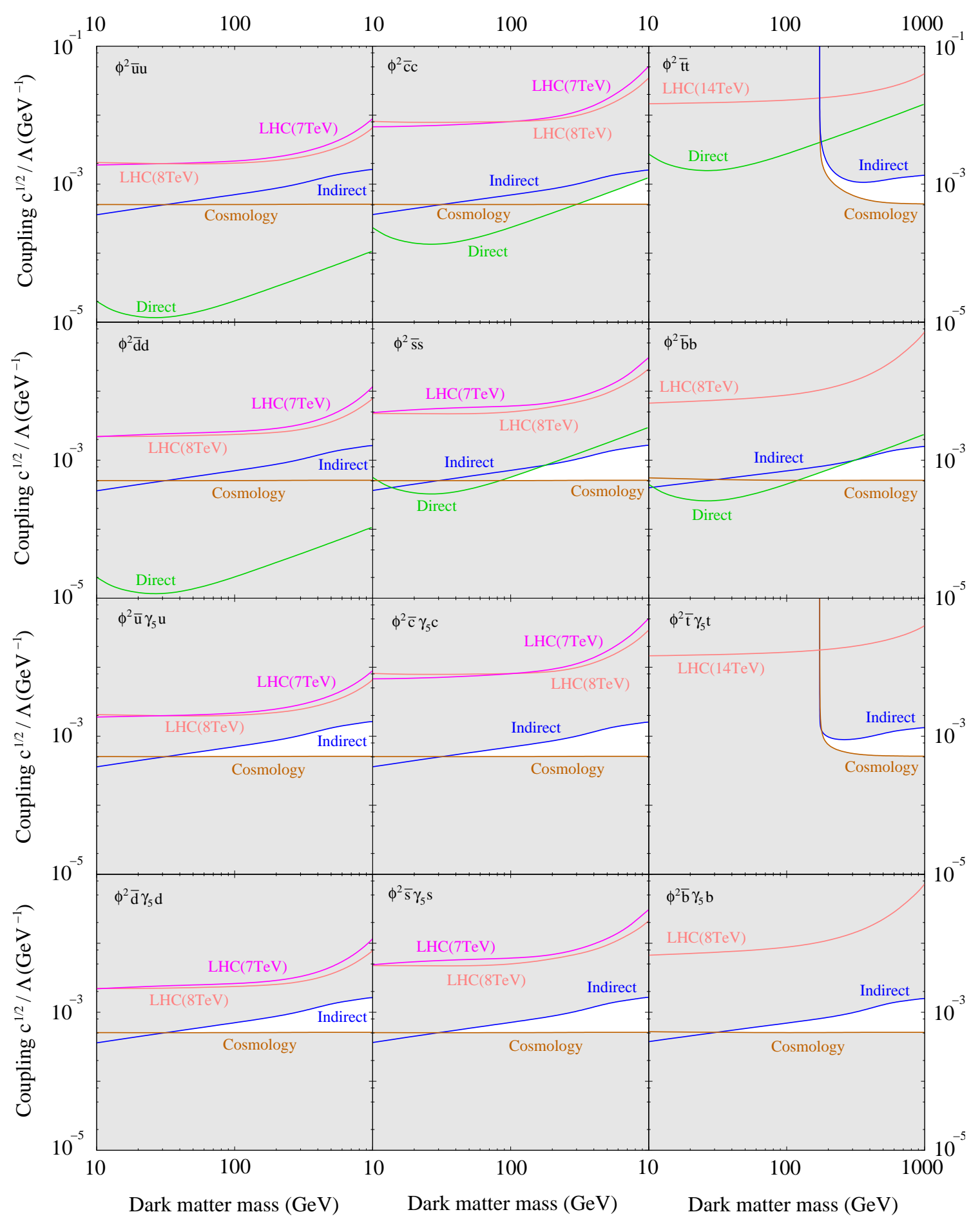

Figure 3: Limits on the operators describing the interactions between the real scalar DM $\phi$ and various quarks. Those from current $\mathrm{LHC}$ ' $\mathrm{LHC}(7 \mathrm{TeV})$ ', future $\mathrm{LHC}$ ' $\mathrm{LHC}(8 \mathrm{TeV})$ \& LHC (14TeV)', cosmological 'Cosmology', direct detection 'Direct', and indirect detection 'Indirect' experiments are shown as magenta, pink, brown, green, and blue lines, respectively in each panel of the figure. 


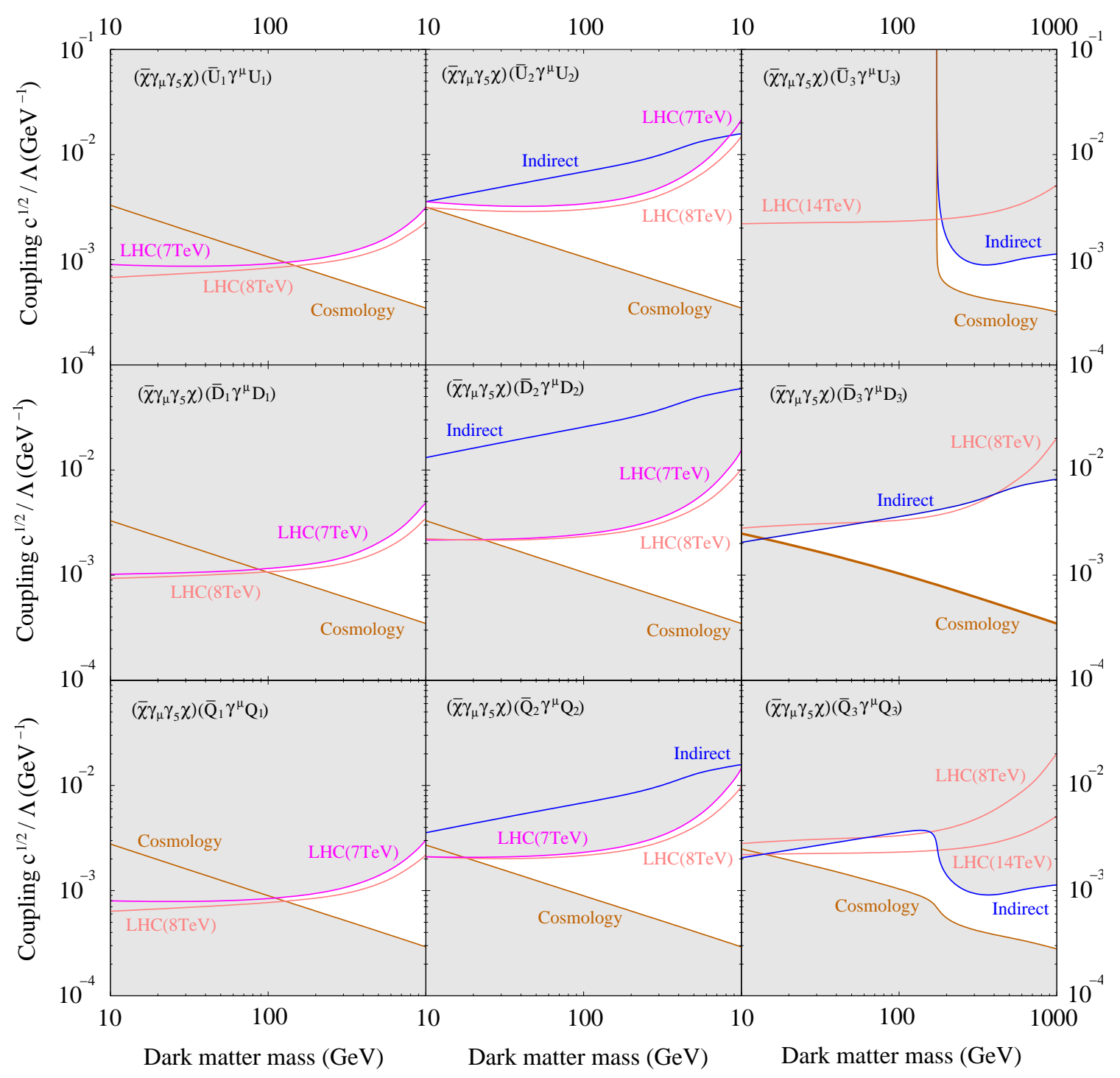

Figure 4: Limits on the operators describing the interaction between the Majorana fermion $D M \chi$ and various quarks. Those from current $\mathrm{LHC}$ ' $\mathrm{LHC}(7 \mathrm{TeV})$ ', future $\mathrm{LHC}$ 'LHC(8TeV) \& LHC (14TeV)', cosmological 'Cosmology', direct detection 'Direct', and indirect detection 'Indirect' experiments are shown as magenta, pink, brown, green, and blue lines, respectively in each panel of the figure. 